\title{
On *-Continuity and *-Uniform Continuity of Some Non-Newtonian Superposition Operators
}

\author{
Fatmanur Erdoğan ${ }^{1 *}$, Birsen Sağır² \\ ${ }^{1 *}$ Ondokuz Mayıs University, Institute of Graduate Education, Department of Mathematics, Samsun,Turkey,(ORCID: 0000-0001-5638-5294), \\ fatmanurkilic89@hotmail.com \\ 2 Ondokuz Mayıs University, Faculty of Science and Arts, Department of Mathematics, Samsun, Turkey, (ORCID: 0000-0001-5954-2005), bduyar@omu.edu.tr
}

(1st International Conference on Applied Engineering and Natural Sciences ICAENS 2021, November 1-3, 2021)

(DOI: 10.31590/ejosat.1012337)

ATIF/REFERENCE: Erdoğan, F. \& Sağır, B. (2021). On *-Continuity and *-Uniform Continuity of Some Non-Newtonian Superposition Operators. European Journal of Science and Technology, (28), 959-967.

\begin{abstract}
Many studies have been done on superposition operators and non-Newtonian calculus from past to present. Sağır and Erdoğan defined Non-Newtonian superposition operators and characterized them on some sequence spaces. Also they examined *- boundedness and *-

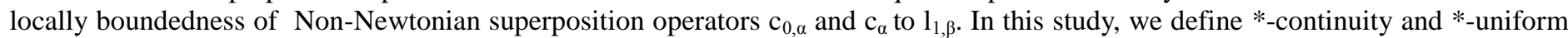
continuity of operator. We have proved that the necessary and sufficient conditions for the *-continuity of the non-Newtonian superposition operator $c_{0, \alpha}$ to $1_{1, \beta}$. Then we examined the relationship between the *-uniform continuity and the *-boundedness of the non-Newtonian superposition operator. Also, the similar results have been researched for the Non-Newtonian superposition operator $\mathrm{c}_{\alpha}$ to $\mathrm{l}_{1, \beta}$.
\end{abstract}

Keywords: *-Continuity, *-uniform continuity, *-boundedness, non-Newtonian superposition operators, non-Newtonian sequence spaces.

\section{Bazı Newtonyen Olmayan Superposition Operatörlerin *-Sürekliliği ve *-Düzgün Sürekliliği Üzerine}

$\ddot{O} z$

Geçmişten günümüze superposition operatörler ve Newtonyen olmayan analiz üzerine birçok çalışma yapılmıştır. Sağır ve Erdoğan Newtonyen olmayan superposition operatörleri tanımlamış ve bazı dizi uzayları üzerinde karakterize etmişlerdir. Ayrıca $\mathrm{c}_{0, \alpha}$ ve $\mathrm{c}_{\alpha}$ uzaylarından $1_{1, \beta}$ uzayına tanımlı Newtonyen olmayan superposition operatörlerin *- sınırlılığını ve *-yerel sınırlılığını incelemişlerdir. Bu çalışmada operatörün *-süreklilik ve *-düzgün sürekliliğini tanımlıyoruz. $\mathrm{c}_{0, \alpha}$ uzayından $1_{1, \beta}$ uzayına tanımlı Newtonyen olmayan superposition operatörün *-sürekliliği için gerekli ve yeterli koşulları ispatlıyoruz. Sonra Newtonyen olmayan superposition operatörün *-düzgün sürekliliği ile *-sınırlılığı arasındaki ilişkiyi inceliyoruz. Ayrıca $\mathrm{c}_{\alpha}$ uzayından $1_{1, \beta}$ uzayına tanımlı Newtonyen olmayan superposition operatör için de benzer sonuçlar araştırılmıştır.

Anahtar Kelimeler: *-Süreklilik, *-düzgün süreklilik, *-sınırlılık, Newtonyen olmayan superposition operatörler, Newtonyen olmayan dizi uzaylar1.

\footnotetext{
*Corresponding Author: fatmanurkilic89@ hotmail.com
} 


\section{Introduction}

Non-Newtonian calculus was firstly introduced by Michael Grossman and Robert Katz between years 1967 and 1970 and they published the book about fundamentals of non-Newtonian calculus which includes some special calculus such as geometric, harmonic, quadratic. At the recent times, Çakmak and Başar (2012) obtained some results on sequence spaces with respect to non-Newtonian calculus. Also, Duyar and Erdogan (2016) worked on non-Newtonian real number series and there are many works about non-Newtonian calculus as (Sağır and Erdoğan, 2019b; Sager and Sağır, 2021).

Many studies are done until today on superposition operator which is one of the non-linear operators. Dedagich and Zabreiko (1987) studied on the superposition operators in the space $\ell_{p}$. After, some properties of superposition operator, such as boundedness, continuity, were studied by Thuangoon (1998), Sağır and Güngör (2015a) and many others (Sağır and Güngör, 2015b; Güngör and Sağır, 2017). Non-Newtonian superposition operator was defined and characterized in some non-Newtonian sequence spaces by Sağır and Erdoğan (2019a). Also Erdoğan and Sağır (2021) worked on $*_{\text {- }}$ boundedness and *-locally boundedness of some non-Newtonian superposition operators.

A generator is defined as an injective function with domain $\mathbb{R}$ and the range of generator is a subset of $\mathbb{R}$. Let take any $\alpha$ generator with range $A=\mathbb{R}_{\alpha}$. Let define $\alpha$ addition, $\alpha$-subtraction, $\alpha$-multiplication, $\alpha$-division and $\alpha$ -order as follows respectively;

$$
\begin{aligned}
& y \dot{+} z=\alpha\left(\alpha^{-1}(y)+\alpha^{-1}(z)\right) \\
& y \dot{-} z=\alpha\left(\alpha^{-1}(y)-\alpha^{-1}(z)\right) \\
& y \dot{\times} z=\alpha\left(\alpha^{-1}(y) \times \alpha^{-1}(z)\right) \\
& y \dot{I} z=\alpha\left(\alpha^{-1}(y) / \alpha^{-1}(z)\right), z \neq \dot{0}, \alpha^{-1}(z) \neq 0 \\
& y \dot{<} z(y \dot{\leq} z) \Leftrightarrow \alpha^{-1}(y)<\alpha^{-1}(z)\left(\alpha^{-1}(y) \leq \alpha^{-1}(z)\right)
\end{aligned}
$$

for $x, y \in \mathbb{R}_{\alpha}$ (Grossman and Katz, 1972).

$\left(\mathbb{R}_{\alpha}, \dot{+}, \dot{x}, \dot{\leq}\right)$ is totally ordered field (Çakmak and Başar, 2012).

$\alpha$-absolute value of a number $x \in \mathbb{R}_{\alpha}$ is defined by

$$
|x|_{\alpha}= \begin{cases}x & , x>\alpha(0) \\ \alpha(0) & , x=\alpha(0) \\ \alpha(0)-x & , x \dot{<} \alpha(0)\end{cases}
$$

Grossman and Katz described the *-calculus with the help of two arbitrary selected generators. In this study, we studied according to $*$-calculus. Let take any generators $\alpha$ and $\beta$ and let $*$ ("star") is shown the ordered pair of arithmetics ( $\alpha$ arithmetic, $\beta$-arithmetic). The following notations will be used. $\alpha$-arithmetic

$\beta$-arithmetic

$\begin{array}{lll}\text { Realm } & A=\mathbb{R}_{\alpha} & B=\mathbb{R}_{\beta} \\ \text { Addition } & \dot{+} & \ddot{+} \\ \text { Subtraction } & \dot{-} & \ddot{-} \\ \text { Multiplication } & \dot{x} & \ddot{x} \\ \text { Division } & \dot{j} & \ddot{j} \\ \text { Order } & \dot{<} & \ddot{<}\end{array}$

In the $*$-calculus, $\alpha$-arithmetic is used on arguments and $\beta$-arithmetic is used on values.

The isomorphism from $\alpha$-arithmetic to $\beta$-arithmetic is the unique function $l$ (iota) that possesses the following three properties.

1. $l$ is one-to-one.

2. $l$ is on $A$ and onto $B$.

3. For any numbers $u$ and $v$ in $A$,

It turns out that $l(x)=\beta\left(\alpha^{-1}(x)\right)$ for every number $x$ in $A$ and that $l(\dot{n})=\ddot{n}$ for every integer $n$ (Grossman and Katz, 1972).

The non-Newtonian sequence spaces $S_{\alpha}, \ell_{\infty, \alpha}, c_{\alpha}$ and $c_{0, \alpha}$ over the non-Newtonian real field $\mathbb{R}_{\alpha}$ are defined as following:

$$
\begin{aligned}
& S_{\alpha}=\left\{x=\left(x_{k}\right): \forall k \in \mathbb{N} ; x_{k} \in \mathbb{R}_{\alpha}\right\} \\
& \left.\ell_{\infty, \alpha}=\left\{x=\left(x_{k}\right) \in S_{\alpha}:{ }^{\alpha} \sup _{k \in \mathbb{N}}\left|x_{k}\right|_{\alpha} \dot{<}\right\}\right\} \\
& c_{\alpha}=\left\{x=\left(x_{k}\right) \in S_{\alpha}: \exists l \in \mathbb{R}_{\alpha}{ }^{\prime}{ }^{\alpha} \lim _{k \rightarrow \infty}\left|x_{k} \dot{-l}\right|_{\alpha}=\dot{0}\right\} \\
& c_{0, \alpha}=\left\{x=\left(x_{k}\right) \in S_{\alpha}:{ }^{\alpha} \lim _{k \rightarrow \infty}\left|x_{k}\right|_{\alpha}=\dot{0}\right\}
\end{aligned}
$$

The sequence spaces $\ell_{\infty, \alpha}, c_{\alpha}, c_{0, \alpha}$ are non-Newtonian normed spaces with the non-Newtonian norm $\|\cdot\|_{\ell_{\infty, \alpha}}$ which is defined as $\|x\|_{\ell_{\infty, \alpha}}={ }^{\alpha} \sup _{k \in \mathbb{N}}\left|x_{k}\right|_{\alpha}$ (Çakmak and Başar, 2012). The $\alpha$-sequence $e_{n}^{(k)}$ is defined as $e_{n}^{(k)}=\left\{\begin{array}{ll}\dot{1}, & k=n \\ \dot{0}, & k \neq n\end{array}\right.$. 
Let $S_{\alpha}$ be space of non-Newtonian real number sequences, $X_{\alpha}$ be a sequence space on $\mathbb{R}_{\alpha}$ and $Y_{\beta}$ be a sequence space on $\mathbb{R}_{\beta}$. A non-Newtonian superposition operator ${ }_{N} P_{f}$ on $X_{\alpha}$ is a mapping from $X_{\alpha}$ into $S_{\alpha}$ defined by ${ }_{N} P_{f}(x)=\left(f\left(k, x_{k}\right)\right)_{k=1}^{\infty}$ where $f: \mathbb{N} \times \mathbb{R}_{\alpha} \rightarrow \mathbb{R}_{\beta}$ satisfies condition $\left(N A_{1}\right)$ as follows;

$\left(N A_{1}\right) \quad f(k, \dot{0})=\ddot{0}$ for all $k \in \mathbb{N}$

If ${ }_{N} P_{f} \in Y_{\beta}$ for all $x=\left(x_{k}\right) \in X_{\alpha}$, we say that ${ }_{N} P_{f}$ acts from $X_{\alpha}$ into $Y_{\beta}$ and write ${ }_{N} P_{f}: X_{\alpha} \rightarrow Y_{\beta}$ (Sağır and Erdoğan, 2019a).

Also, we shall assume the following conditions:

$\left(N A_{2}\right) f(k,$.$) is *$-continuous for all $k \in \mathbb{N}$.

$\left(N A_{2}^{\prime}\right) f(k,$.$) is \beta$-bounded on every $\alpha$-bounded subset of $\mathbb{R}_{\alpha}$ for all $k \in \mathbb{N}$.

Sağır and Erdoğan (2019a) have characterized the nonNewtonian superposition operators ${ }_{N} P_{f}$ on $c_{0, \alpha}$ and $c_{\alpha}$ as the following.

\section{Theorem 1}

Let $f: \mathbb{N} \times \mathbb{R}_{\alpha} \rightarrow \mathbb{R}_{\beta}$ satisfies the condition $\left(N A_{2}^{\prime}\right)$. Then ${ }_{N} P_{f}: c_{0, \alpha} \rightarrow \ell_{1, \beta}$ if and only if there exist an $\alpha$ number $\mu \dot{0} \quad$ and a $\beta$-sequence $\left(c_{k}\right) \in \ell_{1, \beta} \quad$ such that $|f(k, t)|_{\beta} \ddot{\leq} c_{k}$ when $|t|_{\alpha} \dot{\leq} \mu$ for all $k \in \mathbb{N}$.

\section{Theorem 2}

Let $f: \mathbb{N} \times \mathbb{R}_{\alpha} \rightarrow \mathbb{R}_{\beta}$ satisfies the condition $\left(N A_{2}^{\prime}\right)$. Then ${ }_{N} P_{f}: c_{\alpha} \rightarrow \ell_{1, \beta}$ if and only if there exist an $\alpha$ number $\mu \dot{0} \quad$ and a $\beta$-sequence $\left(c_{k}\right) \in \ell_{1, \beta} \quad$ such that $|f(k, t)|_{\beta} \ddot{\leq} c_{k}$ when $|t \cdot z|_{\alpha} \dot{\leq} \mu$ for all $z \in \mathbb{R}_{\alpha}$ and for all $k \in \mathbb{N}$.

\section{Theorem 3}

Let $f: \mathbb{N} \times \mathbb{R}_{\alpha} \rightarrow \mathbb{R}_{\beta}$. The non-Newtonian superposition operator ${ }_{N} P_{f}: c_{0, \alpha} \rightarrow \ell_{1, \beta}$ is $*$-bounded if and only if for all $\mu>\dot{0}$ there exists a $\beta$ sequence $c(\mu)=\left(c_{k}(\mu)\right) \in \ell_{1, \beta}$ such that $|f(k, t)|_{\beta} \ddot{\leq} c_{k}$ when $|t|_{\alpha} \dot{\leq} \mu$ for each $k \in \mathbb{N}$.

\section{Theorem 4}

Let $f: \mathbb{N} \times \mathbb{R}_{\alpha} \rightarrow \mathbb{R}_{\beta}$. Then ${ }_{N} P_{f}: c_{\alpha} \rightarrow \ell_{1, \beta}$ is $*_{-}$ bounded if and only if for every $\mu \dot{>0}$ there exists a $\beta$ e-ISSN: 2148-2683 sequence $c(\mu)=\left(c_{k}(\mu)\right) \in \ell_{1, \beta}$ such that $|f(k, t)|_{\beta} \ddot{\leq} c_{k}$ when $|t|_{\alpha} \dot{\leq} \mu$ for all $k \in \mathbb{N}$ (Erdoğan and Sağır, 2021).

Our aim in this study is to extend some topological properties of superposition operators in classical calculus to nonNewtonian calculus. We prove that the necessary and sufficient conditions for the *-continuity and *-uniform continuity of the non-Newtonian superposition operator ${ }_{N} P_{f}: c_{0, \alpha} \rightarrow \ell_{1, \beta}$. Also the similar results have been obtained for ${ }_{N} P_{f}: c_{\alpha} \rightarrow \ell_{1, \beta}$.

\section{Main Results}

\section{Definition 1}

Let $X \subset \mathbb{R}_{\alpha}$ and let $f: X \rightarrow \mathbb{R}_{\beta}$ be a function. If for every $\beta$-number $\varepsilon \ddot{>} \ddot{0}$, there exists an $\alpha$ number $\delta=\delta(\varepsilon) \dot{>} \dot{0}$ such that

$$
\left|f\left(x_{1}\right) \ddot{-} f\left(x_{2}\right)\right|_{\beta} \ddot{<} \varepsilon \text { when }\left|x_{1} \dot{-} x_{2}\right|_{\alpha} \dot{<} \delta
$$

for all $x_{1}, x_{2} \in X$, then it is said that the function $f$ is $*_{\text {- }}$ uniformly continuous on $X$. The function $f: X \rightarrow \mathbb{R}_{\beta}$ is *continuous if it is *-unifomly continuous.

\section{Definition 2}

Let $\left(X,\|\cdot\|_{X, \alpha}\right)$ and $\left(Y,\|\cdot\|_{Y, \beta}\right)$ be non-Newtonian normed spaces and let $F: X \rightarrow Y$ be an operator. If for every $\beta$-number $\varepsilon \ddot{>} \ddot{0}$, there exists an $\alpha$ number $\delta=\delta(\varepsilon) \dot{>0}$ such that

$$
\left\|F\left(x_{1}\right) \ddot{-} F\left(x_{2}\right)\right\|_{Y, \beta} \ddot{<} \varepsilon \text { when }\left\|x_{1} \dot{-} x_{2}\right\|_{X, \alpha} \dot{<} \delta
$$

for all $x_{1}, x_{2} \in X$, then it is said that the operator $F$ is *uniformly continuous on $X$.

\section{Theorem 5}

Every function which is *-continuous on $\alpha$-closed and $\alpha$ bounded subset of $\mathbb{R}_{\alpha}$ is also *-uniformly continuous on this set.

\section{Proof}

Assume that function $f: X \rightarrow \mathbb{R}_{\beta}$ which is $*_{\text {-continuous }}$ on an $\alpha$-closed and $\alpha$-bounded set $X \subset \mathbb{R}_{\alpha}$ is not *uniformly continuous. Then, there are at least a $\beta$. number $\varepsilon \ddot{>} \ddot{0}$ and $x_{n}, y_{n} \in X$ such that

$$
\left|f\left(x_{n}\right) \ddot{-} f\left(y_{n}\right)\right|_{\beta} \ddot{\geq} \varepsilon
$$

when 


$$
\left|x_{n} \dot{-} y_{n}\right|_{\alpha} \dot{<} \frac{\mathrm{i}}{\dot{n}} \alpha
$$

for all $n \in \mathbb{N}$. The sequence $\left(x_{n}\right)$ is an $\alpha$-bounded sequence since set $X$ is $\alpha$-bounded and $x_{n} \in X$ for all $n \in \mathbb{N}$. By

$\alpha$-Bolzano Weierstrass Theorem (Duyar et al., 2015), $\left(x_{n}\right)$ has an $\alpha$-convergent subsequence $\left(x_{n_{k}}\right)$. Let ${ }^{\alpha} \lim _{k \rightarrow \infty} x_{n_{k}}=x_{0}$. Since $X$ is $\alpha$-closed, $x_{0} \in X$. If we use the inequality 2.2, we get

$$
\begin{aligned}
\left|y_{n_{k}}-x_{0}\right|_{\alpha} & \leq\left|y_{n_{k}}-x_{n_{k}}\right|_{\alpha} \dot{+}\left|x_{n_{k}}-x_{0}\right|_{\alpha} \\
& \dot{\leq} \frac{1}{\dot{n}_{k}} \alpha \dot{+}\left|x_{n_{k}} \dot{-} x_{0}\right|_{\alpha}
\end{aligned}
$$

This means that ${ }^{\alpha} \lim _{k \rightarrow \infty} y_{n_{k}}=x_{0}$. Since function $f$ is $*_{-}$ continuous at point $x_{0}$

$$
{ }^{\beta} \lim _{k \rightarrow \infty} f\left(x_{n_{k}}\right)={ }^{\beta} \lim _{k \rightarrow \infty} f\left(y_{n_{k}}\right)=f\left(x_{0}\right)
$$

is written. Therefore

$$
\left|{ }^{\beta} \lim _{k \rightarrow \infty} f\left(x_{n_{k}}\right) \ddot{{ }^{\beta}} \lim _{k \rightarrow \infty} f\left(y_{n_{k}}\right)\right|_{\beta}=\left|f\left(x_{0}\right) \ddot{-} f\left(x_{0}\right)\right|_{\beta}=\ddot{0}
$$
but this situaiton contradicts with the inequality 2.1 for all $k \in \mathbb{N}$. So the function $f$ is *-uniformly continuous on $X$.

\section{Corollary 1}

All functions which are *-continuous on $[a, b] \subset \mathbb{R}_{\alpha}$ are *-uniformly continuous on this interval.

\section{Proposition 1}

Let $X$ be one of the non-Newtonian sequence spaces $c_{0, \alpha}, c_{\alpha}$ and $\ell_{\infty, \alpha}$. If the non-Newtonian superposition

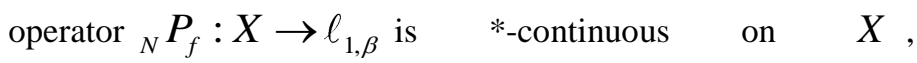
then $f(k,$.$) is { }^{*}$-continuous on $\mathbb{R}_{\alpha}$ for all $k \in \mathbb{N}$.

\section{Proof}

Assume that ${ }_{N} P_{f}$ is $*$-continuous on $X$. Let $k \in \mathbb{N}$, $t_{0} \in \mathbb{R}_{\alpha}$ and $\varepsilon \ddot{>} \ddot{0}$. Since $\quad{ }_{N} P_{f}$ is $*_{\text {-continuous }}$ at $t_{0} \dot{\times} e^{(k)} \in X$, there exists an $\alpha$-number $\delta \dot{>0}$ such that $\left\|{ }_{N} P_{f}(z) \ddot{{ }_{N}} P_{f}\left(t_{0} \dot{x} e^{(k)}\right)\right\|_{\ell_{1, \beta}} \ddot{<} \varepsilon$ when $\left\|z \dot{-} t_{0} \dot{x} e^{(k)}\right\|_{X, \alpha} \dot{<}(2.3)$ for each $z=\left(z_{k}\right) \in X$. Let $t \in \mathbb{R}_{\alpha}$ with $\left|t \dot{-} t_{0}\right|_{\alpha} \dot{<} \delta$ and let $y_{n}=\left\{\begin{array}{l}t, n=k \\ \dot{0}, n \neq k\end{array} . \quad\right.$ Then $y=\left(y_{n}\right) \in X$ and $\left\|y \dot{-} t_{0} \dot{x} e^{(k)}\right\|_{X, \alpha}=\left|t \dot{-} t_{0}\right|_{\alpha} \dot{<} \delta$. By 2.3, it is obtained that $\left\|{ }_{N} P_{f}(y) \ddot{{ }_{N}} P_{f}\left(t_{0} \dot{\times} e^{(k)}\right)\right\|_{\ell_{1, \beta}} \ddot{<} \varepsilon$. Then

$$
\left|f(k, t) \ddot{\sim} f\left(k, t_{0}\right)\right|_{\beta}=\left\|{ }_{N} P_{f}(y) \ddot{{ }_{N}} P_{f}\left(t_{0} \dot{x} e^{(k)}\right)\right\|_{\ell_{1, \beta}} \ddot{<} \varepsilon .
$$

Thus $f(k,$.$) is *-continuous on \mathbb{R}_{\alpha}$ for all $k \in \mathbb{N}$.

\section{Theorem 6}

Let ${ }_{N} P_{f}: c_{0, \alpha} \rightarrow \ell_{1, \beta}$. The non-Newtonian superposition operator ${ }_{N} P_{f}$ is $*_{\text {-continuous on }} c_{0, \alpha}$ if and only if function $f(k,$.$) is *-continuous on \mathbb{R}_{\alpha}$ for all $k \in \mathbb{N}$.

\section{Proof}

First part of theorem (necessity condition) is seen from Proposition 1 . Conversely, assume that function $f(k,$.$) be *-$ continuous on $\quad \mathbb{R}_{\alpha}$ for all $k \in \mathbb{N}$. Let $x=\left(x_{k}\right) \in c_{0, \alpha}$ and $\varepsilon \ddot{>} \ddot{0}$. The function $f$ satisfies condition $\left(N A_{2}^{\prime}\right)$ since it satisfies condition $\left(N A_{2}\right)$. By Theorem 1 , there exist an $\alpha$ number $\mu \dot{>0}$ and a $\beta$-sequence $\left(c_{k}\right) \in \ell_{1, \beta}$ such that

$$
|f(k, t)|_{\beta} \ddot{\leq} c_{k} \quad \text { whenever } \quad|t|_{\alpha} \dot{\leq} \mu
$$

for all $k \in \mathbb{N}$ since ${ }_{N} P_{f}: c_{0, \alpha} \rightarrow \ell_{1, \beta}$. We get

$$
{ }^{\alpha} \lim _{k \rightarrow \infty}\left|x_{k}\right|_{\alpha}=\dot{0} \text { and } \quad \sum_{k=1}^{\infty} c_{k}={ }_{\beta} \sum_{k=1}^{\infty}\left|c_{k}\right|_{\beta} \ddot{<} \ddot{+} \infty
$$

since $x \in c_{0, \alpha}$ and $\left(c_{k}\right) \in \ell_{1, \beta}$. Then, there exists a $N \in \mathbb{N}$ such that

$$
\left|x_{k}\right|_{\alpha} \dot{\leq} \frac{\mu}{\dot{2}} \alpha \text { and }{ }_{\beta} \sum_{k=N}^{\infty} c_{k} \ddot{<} \frac{\varepsilon}{\ddot{3}} \beta
$$

for all $k \geq N$. Thus $\left|x_{k}\right|_{\alpha} \dot{\leq} \mu$ is written for all $k \geq N$. By 2.4, $\left|f\left(k, x_{k}\right)\right|_{\beta} \ddot{\leq} c_{k}$ is obtained for all $k \geq N$. Then

$$
{ }_{\beta} \sum_{k=N}^{\infty}\left|f\left(k, x_{k}\right)\right|_{\beta} \ddot{\leq}_{\beta} \sum_{k=N}^{\infty} c_{k} \ddot{<} \frac{\varepsilon}{\ddot{3}} \beta
$$

is found. Since the function $f(k,$.$) is *-continuous at x_{k}$, there exists an $\alpha$-number $\delta \dot{>0}$ with $\delta \dot{<}^{\alpha} \min \left\{1, \frac{\mu}{\dot{2}} \alpha\right\}$ such that

$\left|f(k, t) \ddot{\sim} f\left(k, x_{k}\right)\right|_{\beta} \ddot{<} \frac{\varepsilon}{\ddot{3} \ddot{\times}(\ddot{N} \ddot{-} \ddot{1})} \beta$ when $\left|t \dot{-} x_{k}\right|_{\alpha} \dot{<} \delta$ 
for $\quad$ all $k \in\{1,2, \ldots, N-1\} \quad$ and $t \in \mathbb{R}_{\alpha}$. Let $\quad$ we take $z=\left(z_{k}\right) \in c_{0, \alpha}$ such that $\|z \dot{-} x\|_{c_{0, \alpha}} \dot{<} \delta$. Then

$$
\left|z_{k} \dot{-} x_{k}\right|_{\alpha} \dot{\leq} \sup _{n}\left|z_{n} \dot{-} x_{n}\right|_{\alpha}=\|z \dot{-x}\|_{c_{0, \alpha}} \dot{<} \delta
$$

for all $k \in \mathbb{N}$. By 2.6

$$
\left|f\left(k, z_{k}\right) \ddot{=} f\left(k, x_{k}\right)\right|_{\beta} \ddot{<} \frac{\varepsilon}{\ddot{3} \ddot{\times}(\ddot{N} \ddot{\sim} \ddot{1})} \beta
$$

is found for $k \in\{1,2, \ldots, N-1\}$. So

$$
{ }_{\beta}^{N-1}\left|f\left(k, z_{k}\right) \ddot{-} f\left(k, x_{k}\right)\right|_{\beta} \ddot{<} \frac{\varepsilon}{\ddot{3}} \beta
$$

is written. Since

$$
\left|z_{k}\right|_{\alpha} \dot{\leq}\left|z_{k} \dot{-} x_{k}\right|_{\alpha} \dot{+}\left|x_{k}\right|_{\alpha} \dot{<} \delta \dot{+} \frac{\mu}{\dot{2}} \alpha \dot{<} \frac{\mu}{\dot{2}} \alpha \dot{+} \frac{\mu}{\dot{2}} \alpha=\mu
$$

for all $k \geq N$, by virtue of 2.4, $\left|f\left(k, z_{k}\right)\right|_{\beta} \ddot{<} c_{k}$ is obtained for all $k \geq N$. Then

$$
\sum_{k=N}^{\infty}\left|f\left(k, z_{k}\right)\right|_{\beta} \ddot{\leq}_{\beta} \sum_{k=N}^{\infty} c_{k} \ddot{<} \frac{\varepsilon}{\dddot{3}} \beta
$$

is written. Therefore

$$
\begin{aligned}
& \left\|{ }_{N} P_{f}(z) \ddot{\sim}_{N} P_{f}(x)\right\|_{\ell_{1, \beta}}={ }_{\beta} \sum_{k=1}^{\infty}\left|f\left(k, z_{k}\right) \because f\left(k, x_{k}\right)\right|_{\beta} \\
& =\sum_{\beta}^{N-1}\left|f\left(k, z_{k}\right) \ddot{=} f\left(k, x_{k}\right)\right|_{\beta} \ddot{t}_{\beta} \sum_{k=N}^{\infty}\left|f\left(k, z_{k}\right) \ddot{f} f\left(k, x_{k}\right)\right|_{\beta} \\
& \left.\check{\check{\Xi}}_{\beta} \sum_{k=1}^{N-1}\left|f\left(k, z_{k}\right)=f\left(k, x_{k}\right)\right|_{\beta} \ddot{+}_{\beta} \sum_{k=N}^{\infty}\left|f\left(k, z_{k}\right)\right|_{\beta} \ddot{+}_{\beta} \sum_{k=N}^{\infty} f\left(k, x_{k}\right)\right|_{\beta} \\
& \ddot{<} \frac{\varepsilon}{3} \beta \ddot{+} \frac{\varepsilon}{3} \beta \ddot{3} \frac{\varepsilon}{3} \beta \\
& =\varepsilon
\end{aligned}
$$

is obtained by using inequalities 2.5, 2.7,2.8. So, the operator ${ }_{N} P_{f}$ is *-continuous on $c_{0, \alpha}$.

\section{Example 1}

Let function $f: \mathbb{N} \times \mathbb{R}_{\alpha} \rightarrow \mathbb{R}_{\beta}$ be defined as $f(k, t)=\frac{|\imath(t) \ddot{\times}(\imath(t) \ddot{*} \ddot{3})|_{\beta}}{\ddot{7}^{k_{\beta}}} \beta . \quad f$ satisfies the condition $\left(N A_{2}^{\prime}\right)$ since $f(k,)$.$* -continuous for all k \in \mathbb{N}$. Let $\mu=\mathrm{i}$ and $t \in \mathbb{R}_{\alpha}$. If $|t|_{\alpha} \dot{\leq} \mathrm{i}$, then

$$
|\imath(t) \ddot{-} \ddot{3}|_{\alpha} \ddot{\leq}|\imath(t)|_{\alpha} \ddot{+} \ddot{3} \ddot{\leq} \ddot{4}
$$

is obtained. So $|f(k, t)|_{\beta}=\frac{|t(t) \ddot{\times}(t(t) \ddot{-} \ddot{3})|_{\beta}}{\ddot{7}^{k_{\beta}}} \beta=\frac{\left.|l(t)|_{\beta} \ddot{x}(t(t) \ddot{3})\right|_{\beta}}{\ddot{7}^{k_{\beta}}} \beta \ddot{\Xi} \frac{\ddot{4}}{\ddot{7}^{k_{\beta}}} \beta$

is found for all $k \in \mathbb{N}$. If $c_{k}=\frac{\ddot{4}}{\ddot{7}^{k_{\beta}}} \beta$ for all $k \in \mathbb{N}$, $\left(c_{k}\right) \in \ell_{1, \beta}$ is obtained. By Theorem 1 , we get that ${ }_{N} P_{f}: c_{0, \alpha} \rightarrow \ell_{1, \beta}$. In view of Theorem $6{ }_{N} P_{f}$ is $*$ continuous on $c_{0, \alpha}$ since function $f(k,$.$) is *-continuous for$ all $k \in \mathbb{N}$.

\section{Theorem 7}

Let ${ }_{N} P_{f}: c_{0, \alpha} \rightarrow \ell_{1, \beta}$. The non-Newtonian superposition operator ${ }_{N} P_{f}$ is *-uniformly continuous on every $\alpha$-bounded subset of $c_{0, \alpha}$ if and only if function $f$ satisfies the condition $\left(N A_{2}\right)$ and the non-Newtonian superposition operator ${ }_{N} P_{f}$ is *-bounded.

\section{Proof}

Assume that ${ }_{N} P_{f}: c_{0, \alpha} \rightarrow \ell_{1, \beta}$ is *-uniformly continuous on every $\alpha$-bounded subset of $c_{0, \alpha}$. By Theorem $6, f(k,)$.$* -$ continuous for all $k \in \mathbb{N}$. Namely, it satisfies the condition $\left(N A_{2}\right)$. Let we take $\rho>\dot{0}$ and $x=\left(x_{k}\right) \in c_{0, \alpha}$ such that $\|x\|_{c_{0, \alpha}} \dot{\leq} \rho$. The function $f$ satisfies the condition $\left(N A_{2}^{\prime}\right)$ since it satisfies the condition $\left(N A_{2}\right)$. By Theorem 1, there exist an $\alpha$-number $\mu \dot{>0}$ and a $\beta$ sequence $\left(c_{k}\right) \in \ell_{1, \beta}$ such that

$$
|f(k, t)|_{\beta} \ddot{\leq} c_{k} \quad \text { whenever } \quad|t|_{\alpha} \dot{\leq} \mu
$$

For all $\quad k \in \mathbb{N}$ since ${ }_{N} P_{f}: c_{0, \alpha} \rightarrow \ell_{1, \beta} \cdot{ }^{\alpha} \lim _{k \rightarrow \infty}\left|x_{k}\right|_{\alpha}=\dot{0}$ since $x \in c_{0, \alpha}$. Then, there exists $\quad N \in \mathbb{N}$ such that $\left|x_{k}\right|_{\alpha} \dot{\leq} \mu$ for all $k \geq N$. By using 2.9, it is obtained that $\left|f\left(k, x_{k}\right)\right|_{\beta} \ddot{\leq} c_{k}$ for all $k \geq N$. Therefore it is written that

$$
{ }_{\beta} \sum_{k=N}^{\infty}\left|f\left(k, x_{k}\right)\right|_{\beta} \ddot{\leq}_{\beta} \sum_{k=N}^{\infty} c_{k} \ddot{\leq}_{\beta} \sum_{k=1}^{\infty}\left|c_{k}\right|_{\beta}=\left\|\left(c_{k}\right)\right\|_{\ell_{1, \beta}} .
$$

Let $m_{k}={ }^{\beta} \sup _{|t|_{\alpha} \leq \rho}|f(k, t)|_{\beta}$ for all $k \in \mathbb{N}$. Since $f$ satisfies the condition $\left(N A_{2}^{\prime}\right), \quad m_{k} \ddot{<} \ddot{+} \infty$ for all $k \in \mathbb{N}$. Since $\|x\|_{c_{0, \alpha}} \dot{\leq} \rho,\left|x_{k}\right|_{\alpha} \dot{\leq} \rho$ for all $k \in \mathbb{N}$. Hence we get that

$$
\left|f\left(k, x_{k}\right)\right|_{\beta} \ddot{\leq} m_{k}
$$


for all $k \in \mathbb{N}$. By 2.10 and 2.11

$$
\begin{aligned}
\left\|{ }_{N} P_{f}(x)\right\|_{\ell_{1, \beta}} & \ddot{\leq}_{\beta} \sum_{k=1}^{N-1}\left|f\left(k, x_{k}\right)\right|_{\beta} \ddot{+}_{\beta} \sum_{k=N}^{\infty}\left|f\left(k, x_{k}\right)\right|_{\beta} \\
& \ddot{\leq}_{\beta} \sum_{k=1}^{N-1} m_{k} \ddot{+}\left\|\left(c_{k}\right)\right\|_{\ell_{1, \beta}} .
\end{aligned}
$$

Then the non-Newtonian superposition operator ${ }_{N} P_{f}$ is $*_{-}$ bounded.

Conversely, assume that $f$ satisfies the condition $\left(\mathrm{NA}_{2}\right)$ and ${ }_{N} P_{f}$ is *-bounded. To show that non-Newtonian superposition operator ${ }_{N} P_{f}$ is *-uniformly continuous on every $\alpha$-bounded subset of $c_{0, \alpha}$, it must be shown that the operator ${ }_{N} P_{f}$ is *-uniformly continuous on $\alpha$-ball $B_{\alpha}[\dot{0}, \rho]$. Let $\rho \dot{>0}$ and $\varepsilon \ddot{>0}$. Since ${ }_{N} P_{f}$ is *-bounded, by Theorem 3 there exists a $\beta$-sequence $c_{k}(\rho) \in \ell_{1, \beta}$ such that

$$
|f(k, t)|_{\beta} \ddot{\leq} c_{k}(\rho) \text { when }|t|_{\alpha} \dot{\leq} \rho
$$

for all $k \in \mathbb{N}$. Since $c_{k}(\rho) \in \ell_{1, \beta}$, there exists a $N \in \mathbb{N}$ such that $\sum_{k=N}^{\infty} c_{k}(\rho) \ddot{<} \frac{\varepsilon}{\ddot{3}} \beta$. Since $f(k,$.$) is *-uniformly$ continuous on $\dot{[} 0 \dot{-} \rho, \rho]$ for all $k \in\{1,2, \ldots, N-1\}$, there exists a $\delta \in \mathbb{R}_{\alpha}$ with $\dot{0} \dot{<} \delta \dot{<} \mathrm{i}$ such that

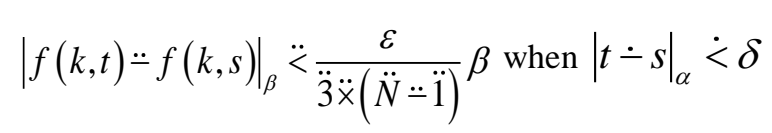

for $t, s \in \dot{[} 0 \dot{-} \rho, \rho \dot{]}$. Let $x=\left(x_{k}\right), \quad y=\left(y_{k}\right) \in B_{\alpha}[\dot{0}, \rho]$ with $\|x \dot{-} y\|_{c_{0, \alpha}} \dot{<} \delta$. Then $\left|x_{k}\right|_{\alpha} \dot{\leq} \rho,\left|y_{k}\right|_{\alpha} \dot{\leq} \rho$ and $\left|x_{k} \dot{-} y_{k}\right|_{\alpha} \dot{<} \delta$ for all $k \in \mathbb{N}$. By inequality 2.13

$$
\left|f\left(k, x_{k}\right) \ddot{\sim} f\left(k, y_{k}\right)\right|_{\beta} \ddot{<} \frac{\varepsilon}{\ddot{3} \ddot{\times}(\ddot{N} \ddot{\sim} \ddot{1})} \beta
$$

is obtained for all $k \in\{1,2, \ldots, N-1\}$. Then

$$
\sum_{\beta=1}^{N-1}\left|f\left(k, x_{k}\right) \ddot{-} f\left(k, y_{k}\right)\right|_{\beta} \ddot{<} \frac{\varepsilon}{\dddot{3}} \beta
$$

$$
\text { is } \quad \text { found. } \quad \text { By 2.12, } \quad\left|f\left(k, x_{k}\right)\right|_{\beta} \ddot{\Xi} c_{k}(\rho)
$$
and $\left|f\left(k, y_{k}\right)\right|_{\beta} \ddot{\leq} c_{k}(\rho)$ are obtained for all $k \in \mathbb{N}$. Therefore

$$
{ }_{\beta} \sum_{k=N}^{\infty}\left|f\left(k, x_{k}\right)\right|_{\beta} \ddot{\leq}_{\beta} \sum_{k=N}^{\infty} c_{k}(\rho) \ddot{<} \frac{\varepsilon}{\dddot{3}} \beta
$$

and

$$
{ }_{\beta} \sum_{k=N}^{\infty}\left|f\left(k, y_{k}\right)\right|_{\beta} \ddot{\leq}_{\beta} \sum_{k=N}^{\infty} c_{k}(\rho) \ddot{<} \frac{\varepsilon}{\ddot{3}} \beta
$$

are written. In view of 2.14, 2.15 and 2.16,

$$
\begin{aligned}
& \left\|{ }_{N} P_{f}(x) \ddot{{ }_{N}} P_{f}(y)\right\|_{\ell, \beta}={ }_{\beta} \sum_{k=1}^{\infty}\left|f\left(k, x_{k}\right) \ddot{-} f\left(k, y_{k}\right)\right|_{\beta} \\
& =\sum_{\beta=1}^{N-1}\left|f\left(k, x_{k}\right) \ddot{f} f\left(k, y_{k}\right)\right|_{\beta} \ddot{+}_{\beta} \sum_{k=N}^{\infty}\left|f\left(k, x_{k}\right) \ddot{-} f\left(k, y_{k}\right)\right|_{\beta} \\
& \ddot{\leq}_{\beta} \sum_{k=1}^{N-1}\left|f\left(k, x_{k}\right) \ddot{f} f\left(k, y_{k}\right)\right|_{\beta} \ddot{t}_{\beta} \sum_{k=N}^{\infty}\left|f\left(k, x_{k}\right)\right|_{\beta} \ddot{+}_{\beta} \sum_{k=N}^{\infty}\left|f\left(k, y_{k}\right)\right|_{\beta} \\
& \ddot{<} \frac{\varepsilon}{3} \beta \ddot{+} \frac{\varepsilon}{3} \beta \ddot{3} \frac{\varepsilon}{\ddot{3}} \beta \\
& =\varepsilon
\end{aligned}
$$

is obtained. Thus the non-Newtonian superposition operator ${ }_{N} P_{f}$ is *-uniformly continuous on every $\alpha$-bounded subset of $c_{0, \alpha}$.

\section{Example2}

Let function $f: \mathbb{N} \times \mathbb{R}_{\alpha} \rightarrow \mathbb{R}_{\beta} \quad$ be defined as $f(k, t)=\frac{|l(t)|_{\beta}}{\ddot{7}^{k_{\beta}}} \beta \quad$ for $\quad$ all $\quad k \in \mathbb{N}$ and $\quad t \in \mathbb{R}_{\alpha}$. Then $f(k,$.$) is *-continuous for all k \in \mathbb{N}$. Let $\mu=\mathrm{i}$ and let $\quad c_{k}=\frac{\dddot{1}}{\dddot{7}^{k_{\beta}}} \beta \quad$ for $\quad$ all $\quad k \in \mathbb{N}$. It is obtained that $f(k, t)=\frac{|l(t)|_{\beta}}{\ddot{7}^{k_{\beta}}} \beta \ddot{\leq} c_{k}$ for all $k \in \mathbb{N}$ and for $t \in \mathbb{R}_{\alpha}$ with $|t|_{\alpha} \dot{\leq} \mu$. Hence we get that ${ }_{N} P_{f}: c_{0, \alpha} \rightarrow \ell_{1, \beta}$ by Theorem 1. Let $\varphi>\dot{0}$ and $t \in \mathbb{R}_{\alpha}$. If $|t|_{\alpha} \dot{\leq} \varphi$ for all $k \in \mathbb{N}$,

$$
|f(k, t)|_{\beta} \ddot{\leq} \frac{l(\varphi)}{\ddot{7}^{k_{\beta}}} \beta
$$

is found. We get $\left(c_{k}(\varphi)\right) \in \ell_{1, \beta}$ if $\left(c_{k}(\varphi)\right)=\left(\frac{l(\varphi)}{\ddot{7}^{k_{\beta}}} \beta\right)$ be taken. Then $|f(k, t)|_{\beta} \ddot{\leq}\left(c_{k}(\varphi)\right)$ is written for all $k \in \mathbb{N}$. By Theorem 3, the non-Newtonian superposition operator ${ }_{N} P_{f}$ is *-bounded. Thus the non-Newtonian superposition operator ${ }_{N} P_{f}$ is *-uniformly continuous on every $\alpha$-bounded subset of $c_{0, \alpha}$ by Theorem 7 .

\section{Theorem 8}

Let non-Newtonian superposition operator ${ }_{N} P_{f}: c_{\alpha} \rightarrow \ell_{1, \beta}$ be given. The non-Newtonian superposition 
operator ${ }_{N} P_{f}$ is $*_{\text {-continuous }}$ on $c_{\alpha}$ if and only if function $f(k,$.$) is *$-continuous on $\mathbb{R}_{\alpha}$ for all $k \in \mathbb{N}$.

\section{Proof}

The necessity condition of theorem is obvious from Proposition 1. Conversely assume that $f(k,$.$) is *-continuous$ on $\mathbb{R}_{\alpha}$ for all $k \in \mathbb{N}$. Let $x=\left(x_{k}\right) \in c_{\alpha}$ and $\beta$ number $\varepsilon \ddot{>0}$ be given. The function $f$ satisfies the condition $\left(N A_{2}^{\prime}\right)$. Since ${ }_{N} P_{f}: c_{\alpha} \rightarrow \ell_{1, \beta}$, there exist an $\alpha$ number $\mu>\dot{0}$ and a $\beta$-sequence $\left(c_{k}\right) \in \ell_{1, \beta}$ corresponding to each $z \in \mathbb{R}_{\alpha}$ such that

$$
|f(k, t)|_{\beta} \ddot{\leq} c_{k} \quad \text { whenever } \quad|t \dot{-}|_{\alpha} \dot{\leq} \mu
$$

for all $k \in \mathbb{N}$ by Theorem 2. Since $x \in c_{\alpha}$, there exists $a \in \mathbb{R}_{\alpha}$ such that ${ }^{\alpha} \lim _{k \rightarrow \infty}\left|x_{k} \dot{-a}\right|_{\alpha}=\dot{0}$. Then there exist an $\alpha$-number $\mu \dot{0} \dot{0}$ and a $\beta$-sequence $\left(c_{k}\right) \in \ell_{1, \beta}$

$$
|f(k, t)|_{\beta} \ddot{\leq} c_{k} \quad \text { when } \quad|t \dot{-} a|_{\alpha} \dot{\leq} \mu
$$

for all $k \in \mathbb{N} \quad$ by 2.17. Since ${ }^{\alpha} \lim _{k \rightarrow \infty}\left|x_{k} \dot{-a}\right|_{\alpha}=\dot{0}$ and $\left(c_{k}\right) \in \ell_{1, \beta}$, there exists $N \in \mathbb{N}$ such that

$$
\left|x_{k}-a\right|_{\alpha} \dot{\leq} \frac{\mu}{\dot{2}} \alpha \quad \text { and } \quad \beta \sum_{k=N}^{\infty} c_{k} \ddot{<} \frac{\varepsilon}{\ddot{3}} \beta
$$

for all $k \geq N$. By 2.18, it is obtained that $\left|f\left(k, x_{k}\right)\right|_{\beta} \ddot{\leq} c_{k}$ for all $k \geq N$. Then

$$
\beta \sum_{k=N}^{\infty}\left|f\left(k, x_{k}\right)\right|_{\beta} \ddot{\leq}_{\beta} \sum_{k=N}^{\infty} c_{k} \ddot{<} \frac{\varepsilon}{\ddot{3}} \beta
$$

is written. Since the function $f(k,$.$) is *$-continuous at $x_{k}$ for all $k \in\{1,2, \ldots, N-1\}$, there exists an $\alpha$-number $\delta \dot{>0}$ with $\delta \dot{<}^{\alpha} \min \left\{\mathrm{i}, \frac{\mu}{\dot{2}} \alpha\right\}$ such that

$\left|f(k, t) \ddot{*} f\left(k, x_{k}\right)\right|_{\beta} \ddot{<} \frac{\varepsilon}{\ddot{3} \ddot{\times}(\ddot{N} \ddot{-1})} \beta$ when $\left|t \dot{1} x_{k}\right|_{\alpha} \dot{<} \delta$

for all $k \in\{1,2, \ldots, N-1\}$ and $t \in \mathbb{R}_{\alpha}$. Let $z \in c_{\alpha}$ be given such that $\|z \dot{-x}\|_{c, \alpha} \dot{<} \delta$. Then $\left|z_{k} \dot{-} x_{k}\right|_{\alpha} \dot{<} \delta$. By 2.20,

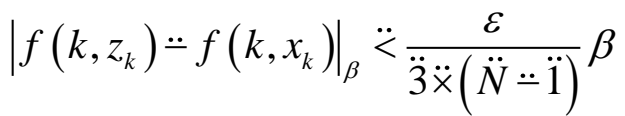

for $k \in\{1,2, \ldots, N-1\}$. From here,

$$
\beta \sum_{k=1}^{N-1}\left|f\left(k, z_{k}\right) \ddot{-} f\left(k, x_{k}\right)\right|_{\beta} \ddot{<} \frac{\varepsilon}{\ddot{3}} \beta
$$

is written. Then

$\left|z_{k} \dot{-a}\right|_{\alpha} \dot{\leq}\left|z_{k} \dot{-} x_{k}\right|_{\alpha} \dot{+}\left|x_{k} \dot{-} a\right|_{\alpha} \dot{<} \delta \dot{+} \frac{\mu}{\dot{2}} \alpha \dot{<} \frac{\mu}{\dot{2}} \alpha \dot{+} \frac{\mu}{\dot{2}} \alpha=\mu$

for all $k \geq N$ and by virtue of $2.18\left|f\left(k, z_{k}\right)\right|_{\beta} \ddot{<} c_{k}$ is obtained for all $k \geq N$. Hence

$$
\beta \sum_{k=N}^{\infty}\left|f\left(k, z_{k}\right)\right|_{\beta} \ddot{\leq} \sum_{k=N}^{\infty} c_{k} \ddot{<} \frac{\varepsilon}{\ddot{3}} \beta
$$

is written. By inequalities 2.19, 2.21 and 2.22,

$$
\begin{aligned}
\left\|N P_{f}(z) \ddot{{ }_{N}} P_{f}(x)\right\|_{\ell_{1, \beta}} & =\sum_{\beta}^{\infty}\left|f\left(k, z_{k}\right) \ddot{=} f\left(k, x_{k}\right)\right|_{\beta} \\
& =\sum_{\beta}^{N-1}\left|f\left(k, z_{k}\right) \ddot{*} f\left(k, x_{k}\right)\right|_{\beta} \ddot{+}_{\beta} \sum_{k=N}^{\infty}\left|f\left(k, z_{k}\right) \ddot{*} f\left(k, x_{k}\right)\right|_{\beta} \\
& \ddot{\leq} \sum_{\beta}^{N-1}\left|f\left(k, z_{k}\right) \ddot{*} f\left(k, x_{k}\right)\right|_{\beta} \ddot{t}_{\beta} \sum_{k=N}^{\infty}\left|f\left(k, z_{k}\right)\right|_{\beta} \ddot{+} \sum_{k=N}^{\infty}\left|f\left(k, x_{k}\right)\right|_{\beta} \\
& \ddot{<} \frac{\varepsilon}{3} \beta \ddot{+} \frac{\varepsilon}{\ddot{3}} \beta \ddot{+} \frac{\varepsilon}{\ddot{3}} \beta \\
& =\varepsilon
\end{aligned}
$$

is obtained. This completes the proof.

\section{Theorem 9}

Let ${ }_{N} P_{f}: c_{\alpha} \rightarrow \ell_{1, \beta}$. The non-Newtonian superposition operator ${ }_{N} P_{f}$ is *-uniformly continuous on every $\alpha$-bounded subset of $c_{\alpha}$ if and only if the function $f$ satisfies the condition $\left(N A_{2}\right)$ and the non-Newtonian superposition operator ${ }_{N} P_{f}$ is *-bounded.

\section{Proof}

Assume that ${ }_{N} P_{f}: c_{\alpha} \rightarrow \ell_{1, \beta}$ is $*_{\text {-uniformly continuous }}$ on every $\alpha$-bounded subset of $c_{\alpha}$. By virtue of Proposition 1 , $f(k,$.$) is *-continuous for all k \in \mathbb{N}$. The function $f$ satisfies the condition $\left(N A_{2}^{\prime}\right)$ since it satisfies the condition $\left(N A_{2}\right)$. Let $\alpha$-number $\gamma \dot{>0}$ and $x=\left(x_{k}\right) \in c_{\alpha}$ be given such that $\|x\|_{c, \alpha} \dot{\leq} \gamma$. By Theorem 2, there exist $\alpha$ number $\mu \dot{0}$ and $\beta$-sequence $\left(c_{k}\right) \in \ell_{1, \beta}$ corresponding to each $z \in \mathbb{R}_{\alpha}$ such that

$$
|f(k, t)|_{\beta} \ddot{\leq} c_{k} \text { when }|t \dot{-}|_{\alpha} \dot{\leq} \mu
$$

for all $k \in \mathbb{N}$ since ${ }_{N} P_{f}: c_{\alpha} \rightarrow \ell_{1, \beta}$. There exists an $a \in \mathbb{R}_{\alpha}$ such that 


$$
{ }^{\alpha} \lim _{k \rightarrow \infty}\left|x_{k} \dot{-a}\right|_{\alpha}=\dot{0}
$$

since $x \in c_{\alpha}$. By 2.23, there exist $\alpha$-number $\mu \dot{0}$ and $\beta$ sequence $\left(c_{k}\right) \in \ell_{1, \beta}$ such that

$$
|f(k, t)|_{\beta} \ddot{\leq} c_{k} \text { when }|t \dot{-a}|_{\alpha} \dot{\leq} \mu .
$$

In view of 2.24 , there exists a $N \in \mathbb{N}$ such that

$$
\left|x_{k} \dot{-} a\right|_{\alpha} \dot{\leq} \mu
$$

for all $k \geq N$. By 2.25 and 2.26, it is said that $\left|f\left(k, x_{k}\right)\right|_{\beta} \ddot{\leq} c_{k}$ for all $k \geq N$. Then

$$
{ }_{\beta} \sum_{k=N}^{\infty}\left|f\left(k, x_{k}\right)\right|_{\beta} \ddot{\leq}_{\beta} \sum_{k=N}^{\infty} c_{k} \ddot{\leq}_{\beta} \sum_{k=1}^{\infty}\left|c_{k}\right|_{\alpha}=\left\|\left(c_{k}\right)\right\|_{\ell_{1, \beta}}
$$

is obtained. Let $m_{k}={ }^{\beta} \sup _{\mid t_{\alpha} \leq \gamma}|f(k, t)|_{\beta}$ for $\quad$ all $k \in \mathbb{N}$. Since $f$ satisfies the condition $\left(N A_{2}^{\prime}\right)$, it is found that $m_{k} \ddot{<} \ddot{+} \infty$ for all $k \in \mathbb{N}$. Since $\|x\|_{c, \alpha} \dot{\leq} \gamma,\left|x_{k}\right|_{\alpha} \dot{\leq} \gamma$ for all $k \in \mathbb{N}$. Hence we get that

$$
\left|f\left(k, x_{k}\right)\right|_{\beta} \ddot{\leq} m_{k}
$$

for all $k \in \mathbb{N}$. By 2.27 and 2.28,

$$
\begin{aligned}
\left\|{ }_{N} P_{f}(x)\right\|_{\ell_{1, \beta}} & =\sum_{\beta}^{N-1}\left|f\left(k, x_{k}\right)\right|_{\beta} \ddot{+}_{\beta} \sum_{k=N}^{\infty}\left|f\left(k, x_{k}\right)\right|_{\beta} \\
& \ddot{\leq} \sum_{k=1}^{N-1} m_{k} \ddot{+}\left\|\left(c_{k}\right)\right\|_{\ell_{1, \beta}}
\end{aligned}
$$

is obtained. Then the non-Newtonian superposition operator ${ }_{N} P_{f}$ is *-bounded.

Conversely, assume that function $f$ satisfies the condition $\left(\mathrm{NA}_{2}\right)$ and the non-Newtonian superposition operator ${ }_{N} P_{f}$ is *-bounded. To show that the non-Newtonian superposition operator ${ }_{N} P_{f}$ is *-uniformly continuous on every $\alpha$-bounded subset of $c_{\alpha}$, it must be shown that ${ }_{N} P_{f}$ is *-uniformly continuous on $\alpha$-ball $B_{\alpha}[\dot{0}, \varphi]$ for all $\varphi \dot{>} \dot{0}$. Let $\varphi \dot{>0}$ and $\varepsilon \ddot{>} \ddot{0}$ be given. Since ${ }_{N} P_{f}$ is *-bounded, by Theorem 4 there exists a $\beta$-sequence $c_{k} \in \ell_{1, \beta}$ such that

$$
|f(k, t)|_{\beta} \ddot{\leq} c_{k} \text { when }|t|_{\alpha} \dot{\leq} \varphi
$$

for all $k \in \mathbb{N}$. Since $c_{k} \in \ell_{1, \beta}$, there exists $N \in \mathbb{N}$ such that ${ }_{\beta} \sum_{k=N}^{\infty} c_{k} \ddot{<} \frac{\varepsilon}{\dddot{3}} \beta$. Since $f(k,$.$) is *-uniformly continuous$ on $[0-\varphi, \varphi]$ for $\quad$ all $k \in\{1,2, \ldots, N-1\}$, there exists a $\delta \in \mathbb{R}_{\alpha}$ with $\dot{0} \dot{<} \delta \dot{<} \mathrm{i}$ such that

$$
|f(k, t) \ddot{*} f(k, s)|_{\beta} \ddot{<} \frac{\varepsilon}{\dddot{3} \ddot{x}(\ddot{N} \ddot{1} \ddot{1})} \beta \text { when }|t \dot{-}|_{\alpha} \dot{<} \delta
$$

for $s, t \in \dot{[} 0 \dot{-} \varphi, \varphi \dot{]}$. Let we take $\quad x=\left(x_{k}\right)$, $y=\left(y_{k}\right) \in B_{\alpha}[\dot{0}, \varphi]$ with $\quad\|x \dot{-} y\|_{c, \alpha} \dot{<} \delta . \quad$ Then $\left|x_{k}\right|_{\alpha} \dot{\leq} \varphi, \quad\left|y_{k}\right|_{\alpha} \dot{\leq} \varphi$ and $\left|x_{k} \dot{-} y_{k}\right|_{\alpha} \dot{<} \delta$ are written for all $k \in \mathbb{N}$. By using inequality 2.30,

$$
\left|f\left(k, x_{k}\right) \ddot{*} f\left(k, y_{k}\right)\right|_{\beta} \ddot{<} \frac{\varepsilon}{\ddot{3} \ddot{\times}(\ddot{N} \ddot{1} \ddot{1})} \beta
$$

is obtained for all $k \in\{1,2, \ldots, N-1\}$. Hence

$$
\sum_{k=1}^{N-1}\left|f\left(k, x_{k}\right) \ddot{\sim} f\left(k, y_{k}\right)\right|_{\beta} \ddot{<} \frac{\varepsilon}{\ddot{3}} \beta
$$

is found. By virtue of $2.31\left|f\left(k, x_{k}\right)\right|_{\beta} \ddot{\leq} c_{k} \quad$ and $\left|f\left(k, y_{k}\right)\right|_{\beta} \ddot{\leq} c_{k}$ are obtained for all $k \in \mathbb{N}$. Therefore

$$
{ }_{\beta} \sum_{k=N}^{\infty}\left|f\left(k, x_{k}\right)\right|_{\beta} \ddot{\leq}_{\beta} \sum_{k=N}^{\infty} c_{k} \ddot{<} \frac{\varepsilon}{\ddot{3}} \beta
$$

and

$$
{ }_{\beta} \sum_{k=N}^{\infty}\left|f\left(k, y_{k}\right)\right|_{\beta} \ddot{\leq}_{\beta} \sum_{k=N}^{\infty} c_{k} \ddot{<} \frac{\varepsilon}{\ddot{3}} \beta
$$

are found. In view of inequalities 2.31, 2.32 and 2.33,

$$
\begin{aligned}
& \left\|{ }_{N} P_{f}(x) \ddot{{ }_{N}} P_{f}(y)\right\|_{c_{1, \beta}}=\sum_{\beta}^{\infty}\left|f\left(k, x_{k}\right) \ddot{*} f\left(k, y_{k}\right)\right|_{\beta} \\
& =\sum_{\beta} \sum_{k=1}^{N-1}\left|f\left(k, x_{k}\right)=f\left(k, y_{k}\right)\right|_{\beta} \ddot{t}_{\beta} \sum_{k=N}^{\infty}\left|f\left(k, x_{k}\right)=\tilde{H} f\left(k, y_{k}\right)\right|_{\beta} \\
& \left.\ddot{\Xi}_{\beta} \sum_{k=1}^{N-1} f\left(k, x_{k}\right) \ddot{*} f\left(k, y_{k}\right)\right|_{\beta} \ddot{t}_{\beta} \sum_{k=N}^{\infty}\left|f\left(k, x_{k}\right)\right|_{\beta} \ddot{t}_{\beta} \sum_{k=N}^{\infty}\left|f\left(k, y_{k}\right)\right|_{\beta} \\
& \ddot{<} \frac{\varepsilon}{3} \beta \ddot{+} \frac{\varepsilon}{\dddot{3}} \beta \ddot{+} \frac{\varepsilon}{3} \beta \\
& =\varepsilon
\end{aligned}
$$

is obtained. Thus the non-Newtonian superposition operator ${ }_{N} P_{f}$ is $*$-uniformly continuous on every $\alpha$-bounded subset of $c_{\alpha}$. 


\section{Results and Discussion}

In this paper we defined *-continuity and *-uniform continuity of operator. We proved that ${ }_{N} P_{f}: c_{0, \alpha} \rightarrow \ell_{1, \beta}$ is *continuous on $c_{0, \alpha}$ if and only if function $f(k,$.$) is *_{\text {- }}$ continuous on $\mathbb{R}_{\alpha}$ for all $k \in \mathbb{N}$. Also we obtained that ${ }_{N} P_{f}: c_{\alpha} \rightarrow \ell_{1, \beta}$ is *-uniformly continuous on every $\alpha$ bounded subset of $c_{\alpha}$ if and only if the function $f$ satisfies the condition $\left(\mathrm{NA}_{2}\right)$ and the non-Newtonian superposition operator ${ }_{N} P_{f}$ is *-bounded.

\section{References}

Grossman, M. And Katz, R. (1972), "Non-Newtonian Calculus", 1st ed., Lee Press, Pigeon Cove Massachussets.

Dedagich, F. and Zabreiko, P.P (1987), "Operator superpositions in the spaces $1_{\mathrm{p}}$ ", Sibirskii Matematicheskii Zhurnal, 28, 86-98.

Thuangoon, A. (1998), "Continuity of superposition operators on some sequence spaces of Maddox", Master of Sciences Dissertation, Graduate School of Chiang Mai University, 66, Thailand.

Çakmak, A.F. and Başar, F. (2012), "Some new results on sequence spaces with respect to non-Newtonian calculus", Journal of Inequalities and Applications, vol. 228, no.1, 1-17.

Duyar, C., Sağır, B. and Oğur, O. (2015), "Some basic topological properties on non- Newtonian real line", British Journal of Mathematics and Computer Science, 9:4, 300307.

Sağır, B. and Güngör, N. (2015a), "Continuity of Superposition Operators on the Double Sequence Spaces $\mathrm{L}_{\mathrm{p}}$ ", Filomat, 9, 2017-2118.

Sağır, B. and Güngör, N. (2015b), "Locally Boundedness and Continuity of Superposition Operators on the Double Sequence Spaces $\mathrm{C}_{\mathrm{r} 0}$ ", Journal of Computational Analysis and Applications, vol. 19(2), 365-377.

Duyar, C. and Erdogan, M. (2016), "On non-Newtonian real number series”, IOSR Journal of Mathematics, vol. 12, iss. 6, ver. IV, 34-48.

Güngör, N. and Sağır, B. (2017), "Continuity of Superposition Operators on Double Sequence Spaces of Maddox $\mathrm{L}_{\mathrm{p}}$ ", Iranian Journal of Science and Technology Transactions A: Science, doi:10.1007/s40995-017-0266-1.

Sağır, B. and Erdoğan, F. (2019a), "On Characterization of NonNewtonian Superposition Operators in Some Sequence Spaces", Filomat, 33:9, 2601-2612.

Sağır, B. and Erdoğan, F. (2019b), "On Function Sequences and Series in the Non-Newtonian Calculus", Journal of Science and Arts, 4(49), 915-936.

Erdoğan, F. and Sağır, B. (2021), "On *- Boundedness and *Local Boundedness of Non-Newtonian Superposition Operators in $c_{0, \alpha}$ and $c_{\alpha}$ to $l_{1, \beta}$ ", Journal of Universal Mathematics, 4(2), 241-251.

Sager, N. and Sağır, B. (2021), "Some inequalities in quasiBanach algebra of non-Newtonian bicomplex numbers", Filomat, 35(7). 